Ann. Zootech., I969, 18 (3), 33I-336.

\title{
UTILISATION PAR LA POULE PONDEUSE DE SUIFS DE DIFFÉRENTES QUALITÉS
}

\author{
B. LECLERCQ et C. JOUANDE'T (*) \\ avec la collaboration technique de M. Bouscaud et L. MARTY \\ Station de Recherches avicoles \\ Centre de Recherches de Tours, 37 - Nouzilly \\ Institut national de la Recherche agronomique
}

\section{SOMMAIRE}

Trois suifs de qualités très différentes (suif "premier jus", suif "qualité volailles" et suif "qualité savonnerie ") ont été incorporés dans des régimes pour poules pondeuses à raison de 5 ou ro $\mathrm{p}$. Ino. Ils sont comparés à un aliment témoin isoénergétique et contenant les mêmes sources de protéines, minéraux et vitamines. L'analyse statistique des résultats ne permet de mettre en éridence aucune différence entre régimes pour toutes les mesures effectuées : taux de ponte, poids moyen de l'wuf, poids total d'wufs pondus, consommation journalière moyenne et indice de consommation.

La qualité des suifs dépend de nombreux facteurs dont les plus importants sont : la nature du produit dont ils sont extraits (tissu adipeux frais ou farine de viande, etc.), le mode d'extraction (centrifugation ou extraction par solvant) et la période de l'année (été ou hiver). Cette qualité est appréciée par des tests physiques et chimiques très divers, dont le plus usité est le taux d'acidité. C'est elle qui détermine à la fois le prix et la destination de cette matière grasse. Étant donné l'intérêt économique de cette source d'énergie pour les régimes des volailles, il nous a semblé important de vérifier dans quelle mesure la qualité peut influencer les performances de ponte.

\section{MATÉRIEL, ET MÉTHODES}

Un troupeau de 23 I poulettes Leghom issues d'un croisement commercial est divisé en 33 blocs de 7 animaux de poids identiques à l'âge de 8 semaines. Ces blocs sont répartis sur trois étages d'une batterie de cages individuelles. La batterie ne comporte qu'une face. On prend soin de disposer à chaque étage les blocs de poicls variés. Les animaux de chaque bloc sont logés côte à côte et les 7 régimes répartis au hasard au sein de chaque bloc.

La composition des régimes figure dans le tableau $\mathbf{I}$. On peut constater qu'une partic commune en constitue la plus grande part $(80 \mathrm{p}$. I00) et assure les apports identiques de proténes, minéraux et vitamines. La fraction résiduelle (20 p. Ioo) caractérise chacun des aliments; elle ne comprend que des aliments non azotés : amidon dans le lot témoin ( $T$ ), suif, amidon et cellulose dans les six autres régimes. Ainsi, d'après le calcul à partir de tables de composition cles aliments, tous les régimes sont isoénergétiques.

* Adresse actuelle : U.C.A. A. B. Chàteau-Thierry-oz. 
Nous donnons dans le tableau 2 les principales caractéristiques des trois suifs employés. Le suif I est un premier jus extrait de tissus adipeux récoltés et préparés le plus tôt possible après l'abattage. Un tel suif est souvent employé en alimentation humaine. Le suif 2 est extrait par

\section{TABLEAU I}

Composition des régimes (en p. roo)

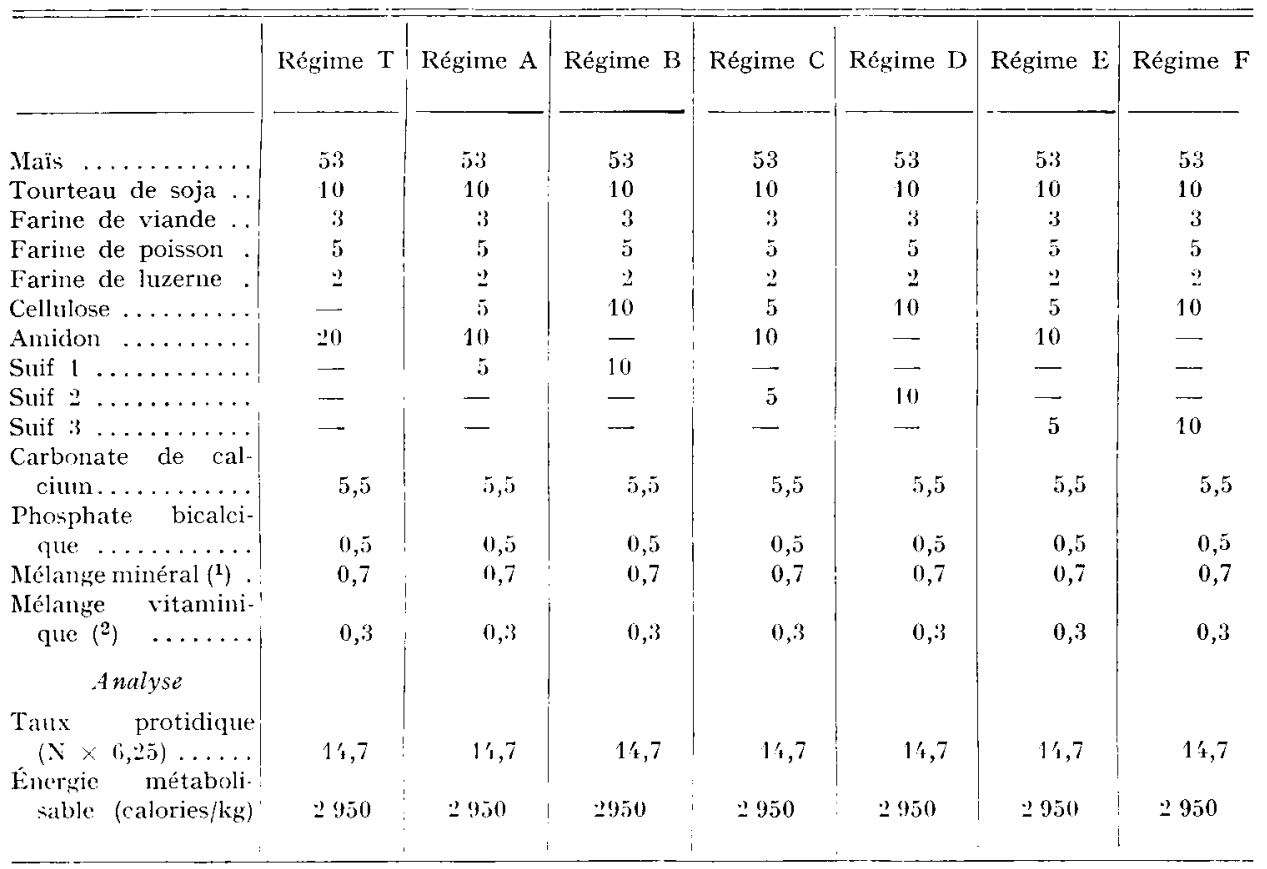

(1) Mélange minéral (pour $100 \mathrm{~kg}$ ) : sulfate de manganèse : $30 \mathrm{~g}$, citrate de fer: $10 \mathrm{~g}$, sulfate de zinc : $1 \mathrm{~g}$, sulfato de cuivre: $0,5 \mathrm{~g}$, iodure de potassium $: 0,5 \mathrm{~g}$, bicarbonate de sodium : $650 \mathrm{~g}$.

(2) Mélange vitaminique : vitamine A : $1000000 \mathrm{UI}$, vitamine D: $100000 \mathrm{UI}$, riboflavine : 0,3 g, pantothénate de calcium : $0,7 \mathrm{~g}$, nicotinamide : $1 \mathrm{~g}$, vitamine $\mathrm{E}: 7,5 \mathrm{~g}$; choline : $56 \mathrm{~g}$, DL méthionine : $100 \mathrm{~g}, \mathrm{BHT}: 10 \mathrm{~g}$, avoine broyée : $56 \mathrm{~g}$.

TABLEAU 2

Caractéristiques des suifs utilisés

\begin{tabular}{|c|c|c|c|}
\hline & Suif 1 & Suif $\Perp$ & Suif 3 \\
\hline Teneur en eau (p. 100).. & 0,068 & 0,130 & 0,460 \\
\hline Acidité oléique (p. 100) . . & 0,31 & $6,4=$ & 26,2 \\
\hline Indice de peroxyde $\ldots .$. & 0,97 & 2,45 & 2,65 \\
\hline $\mathrm{E}_{1}^{1} 270 \ldots \ldots \ldots \ldots \ldots$ & $1,0^{\prime}$ & $1,4^{\prime} \mathbf{t}$ & 2,15 \\
\hline $\mathrm{E}_{1}^{1} 277 \ldots \ldots \ldots \ldots$ & 0,93 & 1,38 & 2,08 \\
\hline $\mathrm{E}_{1}^{1} \geq 81 \ldots \ldots \ldots \ldots \ldots$ & 0,86 & 1,30 & 2,04 \\
\hline $\operatorname{MAT}(\mathrm{N} \times 6,25)(\mathrm{p} .100)$ & 0 & 0,10 & traces \\
\hline
\end{tabular}


centrifugation à partir de farine de viande. Il est généralement destiné à l'alimentation des volailles. Le suif 3 provient également de farines de viande mais il est extrait aux solvants. On le réserve en général à la savonnerie surtout lorsque les farines de viandes ont été conservées dans de mauvaises conditions. Ces 3 suifs diffèrent surtout par leur aciditélibre, leur coefficient d'extinction dans l'UV et le taux des matières azotées entraînées par les solvants. Le tableau 3 permet de comparer les compositions en acides gras des suifs. Le suif 1 se distingue nettement des deux autres par son taux de saturation plus élevé.

TABLEAU 3

Composition en acides gras des suifs utilisés (p. I oo des acides gras dosés)

\begin{tabular}{c|c|c|c}
\hline Acide gras & $\begin{array}{c}\text { Suif } 1 \\
\text { (1 er jus) }\end{array}$ & $\begin{array}{c}\text { Suif 2 } \\
\text { (extraction par } \\
\text { centrifugation) }\end{array}$ & $\begin{array}{c}\text { Suif } 3 \\
\text { (extraction } \\
\text { par solvants) }\end{array}$ \\
\cline { 2 - 3 } $14: 0$ & 4,9 & 2,1 & \\
$16: 0$ & 29,0 & 25,3 & 2,6 \\
$16: 1$ & 2,1 & 4,4 & 26,5 \\
$16: 2 \times 17: 0$ & 1,0 & 1,0 & 4,3 \\
$17: 0$ & 1,0 & 1,4 & 1,0 \\
$18: 0$ & 25,7 & 18,2 & 2,2 \\
$18: 1$ & 34,1 & 43,6 & $4,0,0$ \\
$18: 2$ & 1,9 & 4,2 & 5,0 \\
$18: 3$ & traces & traces & 0,9 \\
\hline
\end{tabular}

La période de ponte est divisée en I I "mois " de 28 jours. Chaque mois, il est déterminé pour chaque animal le nombre total d'œufs pondus, leur poids moyen et la consommation d'aliment. On en déduit l'indice de consommation (consommation d'aliments divisée par le poids d'œufs produits). Nous ne présentons ici que les résultats récapitulatifs de toute la période de ponte.

Pour l'exploitation statistique des résultats, il a été impossible de tenir compte des blocs, du fait de la mortalité qui a réduit à $\mathbf{2} 2$ le nombre de blocs complets. Les animaux ont donc été répartis en deux classes de poids; ceux pesant plus de I $260 \mathrm{~g}$ et ceux pesant moins de I $260 \mathrm{~g}$ lors de la répartition en blocs.

L'analyse statistique permet d'étudier l'effet de "l'étage " dans la batterie, l'effet du poids de la " poule ". l'effet du " régime " ainsi que les interactions entre ces facteurs.

\section{RÉSULTATS}

Dans le tableau 4 nous présentons les résultats de ponte calculés sur des animaux vivant encore en fin d'expérience. Le poids moyen de l'œuf est sensiblement

TABLEAU 4

Résultats de ponte (par poule vivante en fin d'expérience)

\begin{tabular}{|c|c|c|c|c|c|c|c|c|c|}
\hline & Lot $\mathrm{T}$ & Lot A & Lot B & Lot $C$ & Lot D & Lot $\mathrm{E}$ & Lot $F$ & $\begin{array}{l}\text { effet } \\
\text { régime } \\
(\mathrm{F})\end{array}$ & $\begin{array}{l}\text { Signifi- } \\
\text { cation }\end{array}$ \\
\hline Taux de ponte $(\%)$ & 77,1 & 72,7 & 69,6 & 74,5 & 72,9 & 72,8 & 72,5 & $<1$ & NS \\
\hline Poids moyen de l'œuf (g) & 60,1 & 60,5 & 58,9 & 59,4 & 59,5 & 59,8 & $59,1_{1}$ & $<1$ & NS \\
\hline $\begin{array}{l}\text { Poids total d'œuf produit } \\
\quad(\mathrm{kg}) \ldots \ldots \ldots \ldots \ldots \ldots\end{array}$ & 14,24 & 13,55 & 12,61 & 13,60 & 13,33 & 13,60 & 13,25 & 1,95 & NS \\
\hline
\end{tabular}

Annales de Zootechnie. - I969. 
le même dans tous les lots. Des différences légères apparaissent pour le taux de ponte et le poids total d'œuf produit. En moyenne, le lot ' $\mathrm{T}$ apparaît supérieur et le lot $\mathrm{B}$ (Io p. Ioo de suif I) inférieur aux autres lots. Néanmoins, statistiquement ces différences ne sont pas significatives. Seul, l'effet du poids de la " poule " est significatif ; les petites poules pondant des œufs plus petits et un poids total d'œufs plus faible.

Les résultats de consommation journalière d'aliment et l'indice de consommation calculés sur l'ensemble de l'expérience font l'objet du tableau 5 . Là encore on peut remarquer que les valeurs sont très voisines les unes des autres. L'effet du "régime " n'est pas significatif.

TABLEAU 5

Consommation totale d'aliment et indice de consommation

\begin{tabular}{|c|c|c|c|c|c|c|c|c|c|}
\hline & \multirow[b]{2}{*}{ Lot $T$} & \multirow[b]{2}{*}{ Lot $A$} & \multirow[b]{2}{*}{ Lot $B$} & \multirow[b]{2}{*}{ Lot C } & \multirow[b]{2}{*}{ Lot $\mathrm{D}$} & \multirow[b]{2}{*}{ Lot E } & \multirow[b]{2}{*}{ Lot $F$} & \multicolumn{2}{|c|}{ Effet " régime" } \\
\hline & & & & & & & & $\begin{array}{l}\text { Valeur } \\
\text { de F }\end{array}$ & $\begin{array}{l}\text { Signifi- } \\
\text { cation }\end{array}$ \\
\hline $\begin{array}{l}\text { Consommation journa- } \\
\text { lière }(g) \ldots \ldots \ldots \ldots \ldots \\
\text { Indice de consommation }\end{array}$ & $\begin{array}{r}113,5 \\
2,15\end{array}$ & $\begin{array}{c}108,8 \\
2,47\end{array}$ & $\begin{array}{r}103,4 \\
2,52\end{array}$ & $\begin{array}{c}106,1 \\
2,40\end{array}$ & $\begin{array}{r}102,0 \\
2,35\end{array}$ & $\begin{array}{r}106,5 \\
2,45\end{array}$ & $\begin{array}{r}103,6 \\
2,41\end{array}$ & $\begin{array}{l}<1 \\
<1\end{array}$ & $\begin{array}{l}\text { NS } \\
\text { NS }\end{array}$ \\
\hline & & & & & & & & & \\
\hline
\end{tabular}

Nous rapportons à titre indicatif dans le tableau 6 les chiffres de mortalité. Il semble qu'elle soit un peu plus élevée dans les lots recevant du suif. On ne peut cependant en déduire de conclusions valables étant donné le petit effectif utilisé. De plus, il est curieux de constater que ce sont les lots $\mathrm{D}$ et $\mathrm{F}$, ingérant un aliment à ro p. Ioo de suif de mauvaise qualité, qui ont été les moins touchés. On ne pouria conclure sur ce point particulier qu'après des essais portant sur des effectifs beaucoup plus importants.

$$
\text { TABLEAU } 6
$$

Mortalité pendant la période de ponte

\begin{tabular}{|c|c|c|c|c|c|c|c|}
\hline & Lot $\mathrm{T}$ & Lot $A$ & $\operatorname{lot} \mathrm{B}$ & Lot $\mathrm{C}$ & Lot $\mathrm{D}$ & Lot $E$ & Lot $F$ \\
\hline Nombre de poules mortes & 3 & 6 & 8 & 6 & 3 & 7 & 2 \\
\hline Pourcentage de mortalité. & 9 & 18 & $2 / 4$ & 18 & 9 & 21 & 6 \\
\hline
\end{tabular}

Enfin le tableau 7 nous permet de présenter des gains de poids enregistrés pendant la période de ponte. Là encore on ne peut mettre en évidence aucune différence entre les 7 régimes. Ceux qui contiennent ro p. Ioo de suif n'entraînent nullement un engraissement supérieur des pondeuses. 
TABLEAU 7

Gain de poids vif des poules au cours de la période de ponte (en grammes)

\begin{tabular}{|c|c|c|c|c|c|c|c|c|}
\hline Lot & $\mathrm{T}$ & A & I3 & $c^{\prime}$ & $\mathrm{D}$ & E: & $\mathrm{F}$ & Effet Régime \\
\hline Gain de Poids & 546 & 505 & 487 & 555 & 561 & ! & $5^{\prime}+0$ & $F=1,2$ N.S. \\
\hline
\end{tabular}

\section{CONCLUSION}

Avec des souches légères, l'incorporation de suif en quantités importantes dans l'aliment ne présente donc pas d'effets défavorables, dans la mesure tout au moins où le taux énergétique de l'aliment ne s'en trouve pas élevé de façon excessive.

Cette constatation est à rapprocher de celle de FonTAINe et collaborateurs (I967 et I 968) selon lesquelles il est po.ssible d'incorporer jusqu'à I5 p. Ioo de suif dans les aliments pout poules pondeuses sans diminuer les performances de ponte pourvu que la teneur énergétique de l'aliment demeure dans les limites normales. Les effets dépressifs des régimes riches en graisses rapportés par différents auteurs (Doxamisox, I962; WARring et al., I968; Fontaine et al., I967) s'expliquent tous par un niveau excessivement élevé du taux énergétique de l'aliment. Il se traduit par une surconsommation d'énergie, un engraissement important des animaux et des performances de ponte médiocres.

De plus, selon nos résultats, la poule pondeuse semble insensible à la qualité du suif utilisé. Des travaux de Daxgoumau et al. (I962) avaient déjà montré qu'il en est ainsi pour le poulet de chair. Les volailles sont donc d'excellents utilisateurs des suifs de qualité médiocre. Ni leur appétit, ni leurs performances de croissance ou de ponte ne sont diminués par la présence dans leur aliment de suifs jugés de mauvaise qualité d'après les critères classiques.

Rę̧u pour publication on août 1969.

\section{SUMMARY}

DIFFERENT QUALITY FATS USED BY THE LAYING HEN

Three very different quality fats were incorporated into laying hen diets in 5 p. roo or Io $p$. Ioo amounts. These three qualities were represented by fat for human food, fat for animal feeds, and fat for soap-making. These diets were compared to an isoenergetic control feed containing the same protein, mineral and vitamin sources. Statistical analysis did not bring to light any differences in the diets in relation to the following measurements : laying rate, average egg weight total weight of layed eggs, food consumption, and feed efficiency. Incorporating a large quantity of fat into the food of a laying hen thus does not have any unfavorable effects, at least not as long as there is not an excessive amount of energetic constituent in the feed. The laying hen seems unaffected by the quality of fat, and is thus an excellent consumer of mediocre quality fat. 


\section{RÉFÉRENCES BIBLIOGRAPHIQUES}

Dangoumau A., Debru've H., Lavilie A., 1962. Enquête sur les caractères analytiques des matières premières et des produits finis dans l'incorporation des corps gras dans l'alimentation animale. Inds. Alim. Anim., 127, 33-68.

DondLdson W. E., 1962. Effects of type of fat on fat utilization in laying hens. Poult. sci., 41, r640.

Donaldson W. li., I962. Fat tolerance in laying hens. Effects of restricted intake of a high fat diet. Ponlt. Sci., 41, I060-1065.

lontaine G., de Groote G., Reyntwess N., I967. L'influence de rations à valeur énergétique et teneurs en matières grasses différentes, calculées par programmation linéaire, sur la production de pondeuses, type léger, élevées sur litières profondes et en batterie. Rev. de l'A griculture., 3, 435-453.

Fontaine G., Reyntens $\mathfrak{N}$., I968. L'influence des rations à valeur énergétique et teneur en matières grasses différentes, calculées par programmation linéaire, sur la production de pondeuses, type léger, élevées en batteries. Rev. de l'A griculture, 4, 607-618.

Warring J. J., Addison R. 1., JRown. W. O., I968. A comparative study of energy utilization by the laying hen from diets containing a high proportion of fat and diets made up mainly from carbohydrates sources. Br. Poult. Sci., 9, 79-86. 